\title{
SG-TSE: Segment-based Geographic Routing and Traffic Light Scheduling for EV Preemption based Negative Impact Reduction on Normal Traffic
}

\author{
Shridevi Jeevan Kamble, Manjunath R Kounte \\ School of Electronics and Communication \\ REVA University, Bengaluru-560064, India
}

\begin{abstract}
Emergency Vehicles (EVs) play a significant role in giving timely assistance to the general public by saving lives and avoiding property damages. The EV preemption models help the EVs to maintain their speed along their path by pre-clearing the normal vehicles from the path. However, few preemption models are designed in literature, and they lack in minimizing the negative impacts of $\mathrm{EV}$ preemption on normal vehicle traffic and also negative impacts of normal vehicle traffic on EV speed. To accomplish such goals, the work proposes a Segment-based Geographic routing and Traffic light Scheduling based EV preemption (SG-TSE) that incorporates two mechanisms: Segment based Geographic Routing (SGR) and Dynamic Traffic Light Scheduling and EV Preemption (DTSE) for efficient EV preemption. Firstly, the SGR utilized a geographic routing model through the Segment Heads (SHs) along the selected route and passed the EV arrival messages to the traffic light controller to pre-clear the normal traffic. Secondly, the DTSE designs effective scheduling at traffic lights by dynamically adjusting the green time phase based on the minimum detection distance of EVs to the intersections. Thus, the EVs are passed through the intersections quickly without negatively impacting normal traffic, even the signal head in the red phase. Moreover, the proposed SG-TSE activates the green phase time at the correct time and minimizes the negative impacts on the $\mathrm{EV}$ preemption model. Finally, the performance of SG-TSE is evaluated using Network Simulator-2 (NS-2) with different performance metrics and various network traffic scenarios.
\end{abstract}

Keywords-Emergency vehicle (EV) preemption; Segmentbased Geographic routing and Traffic light Scheduling based EV preemption (SG-TSE); geographic routing; Segment based Geographic Routing (SGR); dynamic traffic light scheduling; Dynamic Traffic light Scheduling and EV preemption (DTSE); green phase adjustment

\section{INTRODUCTION}

The Vehicular ad hoc networks (VANETs) enable realtime communication among the roadside vehicles with the support of roadside infrastructure [1]. The VANETs receive high popularity among researchers owing to the application diversity [2]. The VANET applications are mainly categorized into safety and infotainment. In infotainment applications, the vehicles exchange messages about parking areas and hotels and make the journey very comfortable. In contrast to infotainment applications, the safety applications alert the drivers about hazardous situations such as crash warning, accident warning, EV preemption, and others. Hence, the safety applications require strict delay bounds compared with comfort applications. The Emergency Vehicle (EV) preemption is one of the prime VANET applications in which the emergency vehicles are quickly navigated from the approaching lane and intersections [3]. The EVs such as ambulances, fire fighting vehicles, police vehicles, and other defense fighting vehicles receive high priority on roads, as they have to reach their destination on time to save human lives and property losses. The traffic lights integrate various preemption methods and assure desired speed to EVs along its selected path to the incident location to benefit such EVs [4].

The Emergency vehicle preemption system (EVP) interrupts the signal timings of normal traffic at the signalized intersections and provides a green band to the EVs along its routes [5]. Thus, the preemption assists the EVs to pass without stopping or waiting at intersections. It potentially minimizes the travel time and shrinks conflicts with other vehicles in the traveling route [6]. However, it may also negatively impact the general vehicle traffic in the approaching lane. It suffers the vehicles not only in the corresponding intersection but also on other neighboring intersections of coordinated signal control. Hence, it is essential to activate the traffic light green phase at the correct time to reduce the negative impact of EV on normal traffic and also pre-clear the roads in an efficient way to minimize the negative impacts of normal traffic on EVs. Owing to the high dynamic nature and frequent link failure, the geographic routing protocols are highly fit for the VANET environment [7]. Therefore, this work proposes a novel EV preemption model in which segment-based geographic routing and effective traffic light scheduling pass the EVs at intersections quickly with the desired speed.

By designing efficient EV preemption with timely green phase activation, the proposed SG-TSE diminishes both negative impacts, such as the negative impact on normal traffic due to EV preemption and the negative impact on EV due to normal traffic. The conventional methods handle only negative impact issues, resulting in inappropriate $\mathrm{EV}$ preemption and traffic light control. Thus, it leads to losses of human life and property damages. Hence, crucial green phase activation is required with optimal routing strategies. The SETSE solves such an issue significantly by splitting the vehicle density of highly congested scenarios into multiple segments and organizing the vehicles with accurate green phase activation. For that the SG-TSE utilizes a geographic routing 
method. Compared to existing preemption methods, the performance of the proposed model is highly superior in terms of EV preemption speed, especially under a high vehicle density scenario. By navigating the emergency vehicles quickly along its path even the road is congested, the SG-TSE saves human lives and prevents property losses from a hazardous situation.

\section{A. Contribution}

The main contributions of the proposed work are as follows.

- To guarantee the desired speed of EVs at intersections under feasible traffic conditions, this work proposes an SG-TSE protocol that includes two different mechanisms, SGR and DTSE, to achieve its objective.

- To announces the EVs arrival to the traffic light controller, the SGR divides the EV approaching lane into many segments and elects an $\mathrm{SH}$ in each segment for geographic message routing.

- The DTSE uses an effective traffic light scheduling model in which the green phase time is adjusted based on the minimum detection distance of EVs from the intersection that effectively diminishes the normal traffic impacts on EV speed and also EV preemption impact on normal vehicles.

- Finally, the effectiveness of the proposed SG-TSE is evaluated using NS-2. The performance is analyzed with various metrics like packet delivery ratio, overhead, throughput, delay, and EV preemption speed under different network traffic conditions.

\section{B. Paper Organization}

The remaining part of the SG-TSE is organized as follows. Section 2 survey the paper related to EV preemption models and analyzes the gaps in the existing works. Section 3 provides an overview and network model of SG-TSE. Further, it clearly describes the two mechanisms such as SGR and DTSE. Section 4 describes the performance evaluation by applying the simulation parameters and performance metrics for SG-TSE performance analysis. Finally, Section 5 concludes this paper.

\section{LITERATURE SURVEY}

For a clear view, the survey is categorized into two types that are geographic routing methods and $\mathrm{EV}$ preemption methods.

\section{A. Geographic Routing Methods}

A Predictive Geographic Routing Protocol (PGRP) in [8] maximizes the connectivity to deal with VANET dynamicity. The PGRP instructs the vehicles to assign a weight to their neighboring vehicles based on the direction and the angle of the corresponding vehicles. The PGRP can predict the position information of the vehicles at the time with the help of hello packets according to the acceleration information of vehicles. The work in [9] proposes a Maxduration-Minangle GPSR (MM-GPSR) routing protocol that defines a cumulative communication duration in greedy forwarding to obtain the node stability of neighbor nodes. Further, it selects the nodes with maximum cumulative communication duration as nexthop nodes for communication. If the greedy routing is failed, the MM-GPSR utilizes the perimeter mode with the minimum angle method. The node location information is used to estimate the angles. Moreover, the MM-GPSR successfully transmits the packets to the destination with optimal forwarder nodes. The work in [10] proposes a novel geographic routing protocol named Geo-LU to enhance the VANET routing performance. It elaborates the local view of the network topology at the current forwarder by incorporating two-hop neighbor information. It exploits a link utility (LU) measure to measure the utility of a two-hop neighbor link. Further, it takes into account the minimum residual bandwidth on that link and its packet loss rate. Moreover, the Geo-LU effectively reacts to high network traffic and frequent link disconnections by including the two-hop neighbor information with LU measurement. The work in [11] proposes a dissemination mechanism with reroute planning for exchanging the emergency vehicle information.

\section{B. EV Preemption Methods}

Several research works have been designed for emergency vehicle route selection and pre-clearing by integrating the realtime traffic and travel time information [12] [13]. An emergency vehicle pre-emption strategy has been proposed in [14]. Such a preemption model can reduce the delay of emergency vehicle arrival caused due to wide network traffic. It utilizes a connected vehicle infrastructure and efficiently manages the time delay in emergency vehicle arrival. Further, the pre-emption model considers the worst-case nonemergency vehicle's waiting time issues. The work in [15] utilizes an emergency vehicle signal coordination method to offer a green wave to the emergency vehicles. The signal coordination method effectively clears the queue traffic on the road and creates a green phase for quick navigation of emergency vehicles. The work in [16] considers daily emergency vehicle routing issues in a specified network with high spatial resolution and offers effective decision support for emergency vehicular systems. The spatial resolution introduces two advanced technologies that are pre-hospital screening and lane pre-clearing.

The pre-hospital screening offers injury diagnosis of patients and lane pre-clearing assures that the ambulance is moved with desired speed in all lanes. Such a model exploits three various ambulances which can support first aids based on the pre-hospital screening. Moreover, it presents mixedinteger linear programming (MIP) strategy to allocate emergency vehicles to the patient location and navigate the vehicles promptly by planning the shortest traveling routes. Thus, it manages the ambulance fleet properly. A Virtual Traffic Light plus for Emergency Vehicle (VTL+EV) has been proposed in [17] to prioritize the emergency vehicles in an intersection. The VTL+EV is a decentralized and selfcoordinated traffic control system in which the movement of emergency vehicles is expedited, and the normal vehicle waiting time is also minimized.

The work in [18] proposes a Global Positioning System (GPS) based traffic light preemption model to diminish the travel time delay of emergency vehicles. With the GPS data, 
the emergency vehicle can become aware of its position and destination position. The GPS assists the preemption model by incorporating software programs with GPS technology and developing electronic maps to determine the shortest paths. Thus, the emergency vehicle selects the shortest paths based on GPS information and arrives on time. Also, the GPS-based preemption model clears the normal vehicles on the emergency vehicle path by effectively managing the traffic lights of intersections using transmitters. An innovative traffic signal control model in [19] diminishes the response time of emergency vehicles by utilizing connected vehicle infrastructure. Based on the beacons received from an emergency vehicle, such a model instructs the traffic signal to adjust the green phase earlier to reduce the arrival delay of emergency vehicles. The work in [20] proposes a priority signal control algorithm with transit signal priority to improve the emergency vehicle preemption. The transit signal priority model is a proven technique to offer an enhanced public transit operation quality in urban scenarios. The priority model tunes the traffic signal phases based on transit signal priority and serves quick preemptions to an emergency vehicle. Thus, it assists in alleviating the delay in emergency vehicle arrival and minimizing the impact of preemption on general road traffic. An emergency vehicle pre-clearing model in [21] prioritizes the emergency vehicle on the corresponding path by employing the cooperative driving of connected vehicles in a particular area. Such a model converts the connected vehicle cooperative driving issue as a mixed-integer nonlinear programming (MINP) to guarantee the emergency vehicle desired speed and to minimize the impact of pre-emption on connected vehicles. The MINP achieves the objectives by formulating a bi-level optimization model. Initially, the connected vehicles proceeding of the emergency vehicle are divided into various blocks. Further, an emergency vehicle sorting algorithm is applied in each block to sort vehicle trajectories. Thus, the MNP is solved based on the sorting trajectories, and the emergency vehicles are allowed with desired speed on the corresponding path. A novel traffic lightassisted emergency vehicle preemption method at an intersection has been introduced in [22]. Such a model employs wireless vehicles to infrastructure communication among the emergency vehicle and the traffic lights controller for preemption. It estimates the vehicle density at the intersections based on the messages and builds a dynamic mathematical model to discharge the vehicles in the queue.

The work in [23] utilizes a multi-objective programming model for emergency vehicle pre-emption at intersections. The main intention of such a model is to clear the emergency vehicles quickly at the intersection and increase the passing rate of normal vehicles by minimizing the emergency vehicle preemption impact. The work in [24] mainly focuses on constructing better routes for emergency vehicles by designing a realistic traffic-based optimization model. It obtains realtime traffic knowledge from the Google Maps Distance Matrix API. Finally, it finds the best shortest emergency vehicle path with less congestion. The real-time traffic flow-based dynamic and efficient traffic light scheduling algorithm in [25] adjusts the finest green phase time at the signalized road intersection based on realistic traffic information. It also considers the emergency vehicle presence in green phase time adjustment and assists for quick emergency vehicle passing. A multiagent preemptive longest queue first system has been proposed in [26] to handle the emergency vehicle crossings at interrupted intersections. Further, an efficient preemption strategy is selected to diminish the negative impact of preemption on general traffic in [27]. It utilizes the VANET communication through the emergency vehicle path. Thus, it clears the entire route of an emergency vehicle in advance without disturbing the normal traffic flow. The work in [28] utilizes the internet of things technology to facilitate emergency vehicles crossing the intersections quickly. Such a model gathers the EV data along its route periodically and intermittently and provides high priority to the EVs, especially at intersections. A signal priority algorithm in [33] develops a queue length-based green signal activation model in which the signal green phase is extended to the specific road that experiences a high delay. The priority algorithm considers queue length to solve the arrival time issues of the emergency vehicle and reduce the impact on normal vehicles along the emergency vehicle route. The smart emergency vehicle plan model in [30] designs an efficient EV communication model by utilizing app monitoring and a centralized network. The vehicles in the traffic control system have a unique identity number to establish a connection with a centralized server around the traffic signal. The centralized network maintains the vehicular network data, and it plans effective routes to the emergency vehicles. A novel EV preemption method in [31] exploits the advantage of the vehicle to infrastructure communication and vehicle density queue information to manage the traffic light controller. However, it lacks to consider the negative impact of EV preemption on normal traffic.

\section{Research Gap and Problem Statement}

Numerous emergency vehicle preemption and route selection methods are designed in the existing literature to pass the emergency vehicles quickly to the destination. Most of the emergency vehicle route selection model considers the traffic congestion and route length in the emergency vehicle path discovering. However, an emergency vehicle may be delayed due to the signalized intersections along its selected shortest path in urban scenarios. A minute of emergency vehicle delay causes tremendous loss of lives, and hence, it is crucial to minimize the impact of intersection delay caused due to inefficient traffic light scheduling. With aiming to solve such issues, the later researches utilize efficient preemption methods in which the vehicle and infrastructure communication are used to clear the emergency vehicle path or lane in an advanced manner. Such models allow the emergency vehicles to take high priority at the intersections even the signal is in the red phase. In such situations, there is a chance of accidents due to inexperienced and careless driver behaviors. Therefore, it is crucial to activating the signal preemption at the right time using appropriate scheduling methods. The proposed work attempts to design an efficient emergency vehicle preemption model in which geographic routing and timely traffic light scheduling are used to quickly navigate the emergency vehicles and reduce the impact of normal traffic. 


\section{DESIGN Overview OF SG-TSE}

Generally, a less congested route with a short travel time is suggested for emergency vehicles. However, the suggested best routes may be suffered by normal vehicle traffic flows and the hindrances of normal road topology. This work proposes an emergency vehicle pre-emption method with the assistance of vehicular geographic routing and traffic light scheduling to reduce the negative impacts of normal vehicle traffic on emergency vehicle speed. Fig. 1 shows the block diagram of the proposed methodology. Initially, the traffic light and RSU detect the EV on the selected less congested and shortest traveling route based on the routing messages disseminated by EV. The disseminated messages include information about EV presence and speed. Thus, the EV is detected based on the messages. The pre-emption distance measurement is applied to compute the distance between the $\mathrm{EV}$ and road intersections in which the normal vehicle traffic is high. Secondly, the multi-criteria-based preclearance is utilized to clear the normal vehicles quickly in the approaching route and minimizes the disturbances associated with emergency vehicle speed. Further, the normal vehicles are cleared rapidly from the approaching route based on green phase adjustment. Moreover, the proposed methodology minimizes the negative impacts on emergency vehicle speed and provides timely help to the public.

\section{A. Network Model}

The vehicular network is modeled as a communication graph $\mathrm{G}(\mathrm{N}, \mathrm{E})$, where $\mathrm{N}$ refers to the number of nodes classified into emergency vehicles, non-emergency vehicles, RSUs, and traffic light controllers. It is assumed that the virtual traffic lights are installed at every intersection, referred to as RSUs. The term $\mathrm{N}$ refers to the communication link between any two entities. The vehicles in SG-TSE move with the desired speed $\mathrm{S}$. The speed of EV is high than normal vehicles $S_{\mathrm{EV}}>\mathrm{S}_{\mathrm{NV}}$. The $\mathrm{EV}$ does not change its speed along its path using geographic routing and traffic light schedulingbased preemption. Every vehicle in the SG-TSE is equipped with GPS, and it updates its location itself. The other vehicles knew the location of emergency vehicles based on dissemination messages. The vehicles are also equipped with On-Board Units (OBUs) for enabling wireless communication. The emergency vehicles disseminate the beacons to the others in the corresponding segment by using OBUs. Each vehicle has a road map for path selection. The shortest and less congested traveling route of EV is suffered by normal network traffic in urban scenarios. To reduce the negative impact of normal vehicles on the EV route, the SGTSE divides the EV approaching lane into multiple segments $\mathrm{S}=\left\{\mathrm{S}_{1}, \mathrm{~S}_{2}, \ldots . . \mathrm{S}_{\mathrm{n}}\right\}$. In each segment, a Segment Head (SH) is selected for centric-based geographic routing. Further, the signal head green phase adjust time $\mathrm{t}_{\mathrm{g}}$ is computed using the minimum detection distance metric.

\section{B. Segment based Geographic Routing (SGR)}

Initially, the emergency vehicle selects the best traveling route with minimum congestion and short travel time [32]. However, the shortest route includes some normal traffic that influences the desired speed of emergency vehicles. Therefore, it is essential to create an alert about the emergency vehicle arrival to reduce the negative impacts of normal vehicles. The TG-TSE utilizes the SGR to inform the emergency vehicle presence and speed to the traffic controllers along its selected path. The SGR exploits segments to accomplish information dissemination segment-based routing. The SGR routing decision depends on vehicle location information, direction, vehicle density, and link quality among the two communicating parties. Initially, the SGR separates the selection path into multiple segments and inaugurates the geographic routing through the head node elected in each segment. The SGR routing decision is based on the segment data with a look ahead of the next segment data. The message is forwarded through the segment heads until it reaches the traffic controller.

Segment Formation: The SGR is based on various routing parameters like location information, direction, link quality, and traffic density of road networks. The main intention of SGR is to quickly inform about its presence to the traffic controller for efficient preemption. The EV routes the messages in two ways. Firstly, the EV straightly informs the traffic controller when the EV and traffic controller is in the same segment. Otherwise, the EV divides the corresponding path into multiple segments based on the location information and road map data for multi-hop forwarding. In Fig. 2, the segment formation of the proposed SGR is depicted. The SGR forms the segments based on the road trajectory and the number of intersections of the EV vehicle path [29].

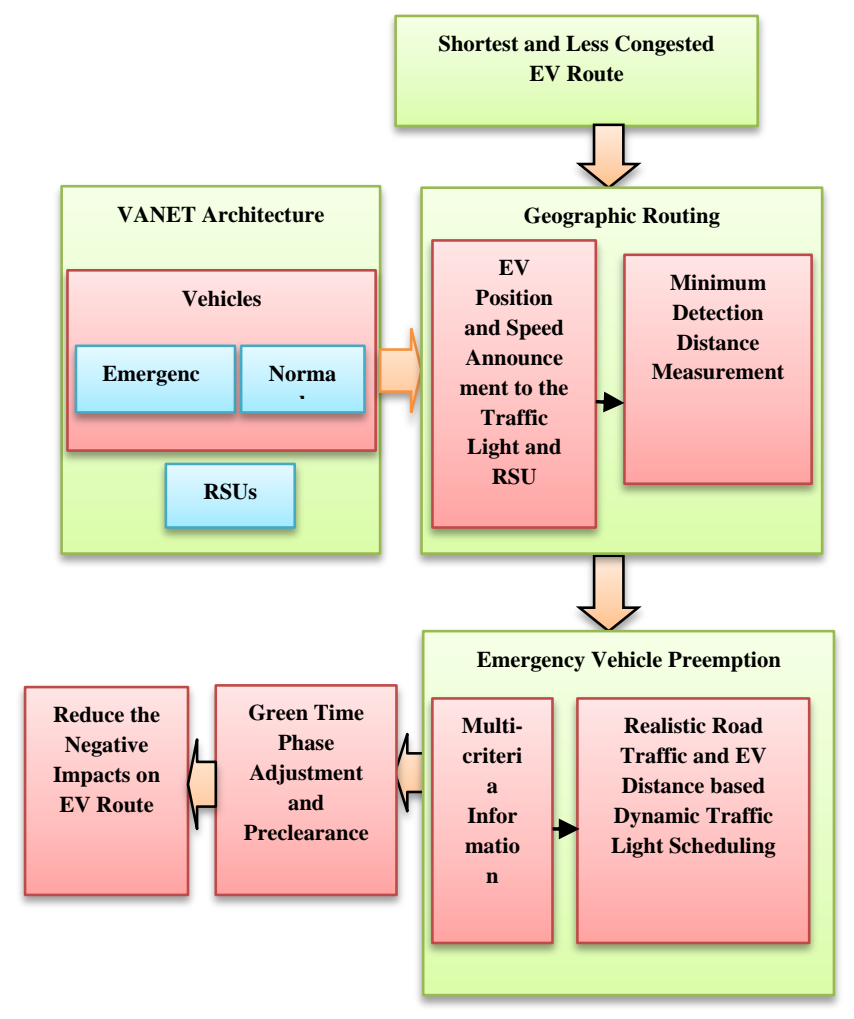

Fig. 1. Block Diagram of Proposed Methodology. 


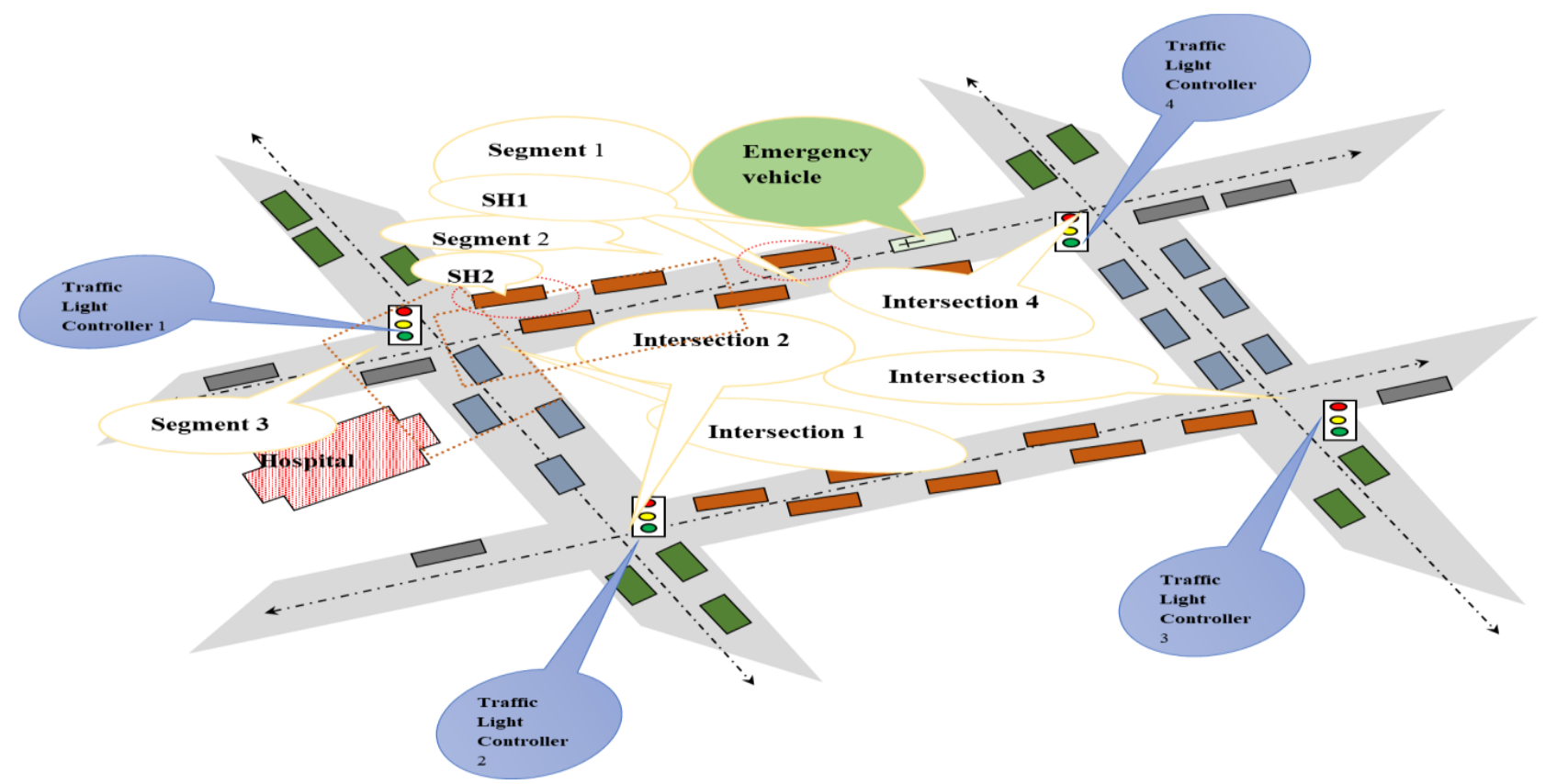

Fig. 2. SGR Segment Formation and Routing.

Segment Head Selection: The segment head is a crucial forwarding node in SGR, as the emergency vehicle arrival is informed through the head to the traffic light controller for preemption. The segment head (SH) selection is initiated after segment formation. Every vehicle announces its position, speed, direction, and link quality through its beacon messages in VANETs. The segment centric distance of a node $D(c)$ is measured using the location information of the node $n$ at $t$ and $t+1$ time intervals. It is estimated as follows.

$\mathrm{D}(\mathrm{c})=\mathrm{L}(\mathrm{t}+1)-\mathrm{L}(\mathrm{t})$

The terms $L(t+1)$ and $L(t)$ are location coordinates of $(\mathrm{x} 2, \mathrm{y} 2)$ and $(\mathrm{x} 1, \mathrm{y} 1)$, at $\mathrm{t}+1$ and $\mathrm{t}$ time, respectively. Further, the SGR utilizes the Pythagoras theorem to estimate the location of the SH node, as depicted in equation (2).

$\mathrm{D}_{\mathrm{c} \rightarrow \mathrm{n}}=\sqrt{\left(\mathrm{x}_{2}-\mathrm{x}_{1}\right)^{2}+\left(\mathrm{y}_{2}-\mathrm{y}_{1}\right)^{2}}$

More than one nodes are presented near the centric location. Therefore, the SGR considers the link quality and direction parameters in SH selection to speed up the message delivery rate without compromising the reliability. Consequently, the SGR computes a score value for the nodes that are suitable for SH. The first metric is location information, in which the node should have to present near or exactly in the central position of the segments. Further, the progressive distance towards the destination is an essential routing metric in which the vehicles are moving in the same direction of $\mathrm{EV}$ is selected as $\mathrm{SH}$. The direction difference score is estimated using the following equation.

$S_{\mathrm{DD}}=\left\{\begin{array}{c}1 ; \text { Same Direction } \\ 0 ; \text { Opposite Direction }\end{array}\right.$

Finally, the SGR computes the overall score value of the SH nodes using the following equation (4).

$\mathrm{S}_{\mathrm{SH}}=\alpha * \mathrm{D}_{\mathrm{c} \rightarrow \mathrm{n}}+\beta * \mathrm{~S}_{\mathrm{DD}}+\gamma * \mathrm{~L}_{\mathrm{Q}}+\varphi * \mathrm{~S}_{\mathrm{v}}$
In equation (4), the terms $D_{c \rightarrow n}, S_{D D}, L_{Q}$ snd $S_{v}$ are the distance, direction, link quality, and speed values of the vehicle suitable for SH. The terms $\alpha, \beta, \gamma$, and $\varphi$ are weighting factors. The summation of weighting factors is equal to 1 . Finally, the SGR selects the node that has high $\mathrm{S}_{\mathrm{SH}}$ value as $\mathrm{SH}$. This process is performed in all the segments of the EV traveling path. Further, the EV informs the traffic controller about the arrival through the selected $\mathrm{SH}$ vehicles.

\section{Dynamic Traffic Light Scheduling and EV Preemption (DTSE)}

After receiving the EV arrival information, the traffic light controller in SG-TSE initiates the traffic light scheduling process DTSE. The traffic light controller has to identify the $\mathrm{EV}$ in advance with a minimum detection time to pre-clear the approaching lane before the EV arrival and neglect the EV waiting time at the intersection. Hence, efficient scheduling is essential to minimize the impact of the normal vehicle on EV preemption. For traffic light scheduling, the DTSE uses the minimum detection distance metric, which is a type of distance measurement applied between the emergency vehicle location and the traffic light intersection. The controller receives the location and speed information of EV from the SH node, and it starts to calculate the minimum detection distance between the EV from the intersection using equation (5).

$\mathrm{t} \geq \mathrm{t}_{\text {switchover }}+\mathrm{t}_{\min }+\mathrm{STI}$

Where the term $t_{\text {switchover }}$ is the switchover time of the signal head and the term $t_{\min }$ is the discharge time of the signal. The term STI is the safety time interval to pre-clear the vehicles in the approaching lane of EV. The switchover time is the interval of switching the signal state. The discharge time $t_{\min }$ is estimated from the average queue length and the queue discharge speed of the EV approach using historical information. The STI value is kept at the constant of $2 \mathrm{~s}$. The $\mathrm{t}$ 
value and speed information are used to calculate the minimum detection time. Thus, the TSE preemption method inaugurates the preemption phase at the correct time using minimum detection distance. Thus, the TSE avoids the hindrances of EV traveling path and minimizes the impact of the preemption on normal vehicular traffic. Further, the TSE starts the green phase adjustment for quick EV navigation through the corresponding intersection.

1) Green phase adjustment: Based on the distance measurement and multiple criteria like congestions and road conditions, the EV preemption is timely activated in the SGTSE. A significant parameter is the signal head green time requirement $\left(t_{g}\right)$ on the $\mathrm{EV}$ preemption, which is computed as follows.

$\mathrm{t}_{\mathrm{g}}=\mathrm{t}-\left(\mathrm{t}_{\text {switchover }}+\mathrm{t}_{\min }+\mathrm{STI}\right)$

By using the $t_{g}$ value, the SG-TSE effectively pre-clears the vehicles in the EV approaching lane and assists the EVs to maintain their desired speed in the corresponding intersection. In the coordinated route intersections case, the TSE assumes that the distance between two successive intersections is smaller than the detection time of EV detection distance. The $\mathrm{EV}$ detection point suffers the first intersection. To rectify such an issue, the SG-TSE considers the notification period of the discharge time of both consecutive intersections. In other words, the SG-TSE treats the two consecutive intersections as a single intersection. Otherwise, the distance between the two consecutive intersections is high than the minimum detection distance, and the SG-TSE re-estimates the detection distance for the second intersection by using the TSE model for preemption. Moreover, the green phase of the traffic light is scheduled at the correct time according to the emergency vehicle distance from the intersection. The SG-TSE protocol process is explained in algorithm 1.

Algorithm. 1. SG-TSE Protocol Process
//SG-TSE Protocol Process//
Input: Selected less congested and shortest EV travelling route
Methods: SGR and DTSE
Output: EV preemption
SG-TSE Do \{
Inputs the EV travelling route and initializes the network;
Starts the SGR and DTSE;
SGR Do \{
Initiates the segment formation for geographic routing;
Divides the approaching route into multiple segments;
Elects an SH in each segment based on a multi-criteria value;
Passes the EV arrival messages to the traffic light controller
\}
through SHs;
DTSE Do \{
Measures the minimum detection distance using equation (5);
Initiates green phase adjustment;
Calculates signal head green time requirement ( $\mathrm{t}_{\mathrm{g}}$ );
Switchovers the green phase at right time;
Pre-clears the EV route;
EV preemption;
\}\};

\section{Performance Evaluation}

The SG-TSE performance is analyzed using NS-2. The performance of the proposed work is compared with existing MDRP [11], PGRP [8], MM-GPSR [9], and Geo-LU [10] for performance evaluation. The simulation parameters are demonstrated in Table I.

TABLE I. SimULATION PARAMETERS

\begin{tabular}{|l|l|}
\hline Parameter & Value \\
\hline Simulation Tool & NS-2 \\
\hline Network Area & $1000 \times 1000$ \\
\hline Number of Nodes & 10 to 30 \\
\hline Number of EVs & $1-3$ \\
\hline Vehicle Communication Range & $50 \mathrm{~m}$ \\
\hline RSU Communication Range & $250 \mathrm{~m}$ \\
\hline Routing Protocol & SG-TSE \\
\hline Traffic Simulator & SUMO \\
\hline Transport Protocol & UDP, CTP \\
\hline Speed of Normal vehicles & $45 \mathrm{Km} / \mathrm{hr}$ \\
\hline Speed of EVs & $60 \mathrm{Km} / \mathrm{hr}$ \\
\hline Propagation Model & Two Ray Ground \\
\hline Simulation Time & 2 Seconds \\
\hline Application Type & CBR \\
\hline Packet Size & 128 Bytes \\
\hline Data Rate & $3 \mathrm{Mbps}$ \\
\hline
\end{tabular}

A. Performance Metrics

The efficacy of SG-TSE is analyzed in terms of packet delivery ratio, overhead, throughput, delay, and EV preemption speed.

- Packet Delivery Ratio (PDR): It is the percentage of successfully delivered packets to the total number of generated packets.

- Overhead: It is the number of extra packets used to perform network operations.

- Throughput: It is the rate of data delivery.

- Delay: It is the time taken to deliver a packet from a source to a destination.

- EV preemption Speed: It is the speed maintained by the EVs on the approaching lane.

\section{B. Simulation Results}

Fig. 3 portrays the comparative PDR results of SG-TSE, MDRP, PGRP, MM-GPSR, and Geo-LU observed under different node density scenarios. From the results of Fig. 3, the SG-TSE increases the PDR from vehicle density 10 to 20, whereas the PDR is decreased after the point of 20 node density scenario. It is caused due to the adequate number of vehicles offer better connectivity to packet forwarding, and the high number of nodes competes to access similar links, resulting in some packet loss in the network. For example, the 
SG-TSE accomplishes 99\%, 99.6\%, and 98\% of PDR for 10, 20, and 30 node densities, respectively. However, the PDR of SG-TSE is higher than the other four geographic routing protocols from 10 to 25 node densities. For instance, the SGTSE improves the PDR by $1.4 \%, 3 \%, 1.9 \%$, and $1.5 \%$ than the existing MDRP, PGRP, MM-GPSR, and Geo-LU protocols, respectively, when 10 nodes are present in the network. The figure shows that the SG-TSE and Geo-LU accomplish 98\% and $99.4 \%$ of PDR in 30 node scenarios. The link utility aware geographic router node selection in Geo-LU improves the PDR than the proposed SG-TSE. However, the SG-TSE attains better PDR values than the MEDRP, PGRP, and MMGPSR protocols, when 30 numbers of vehicles are presented in the network.

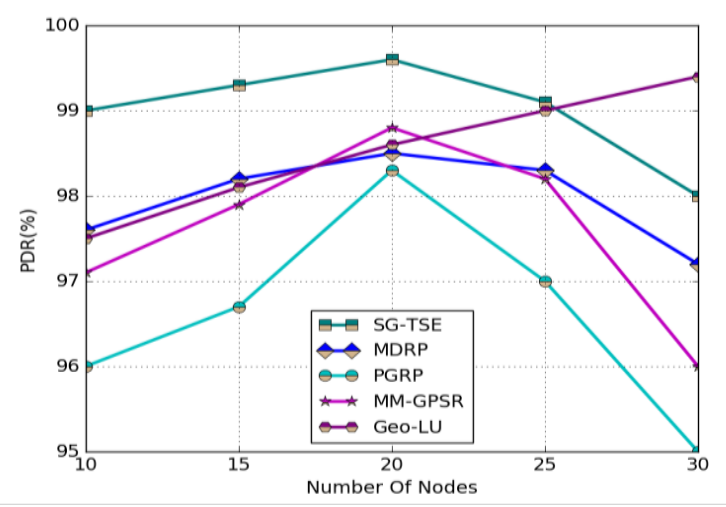

Fig. 3. Number of Nodes vs. PDR.

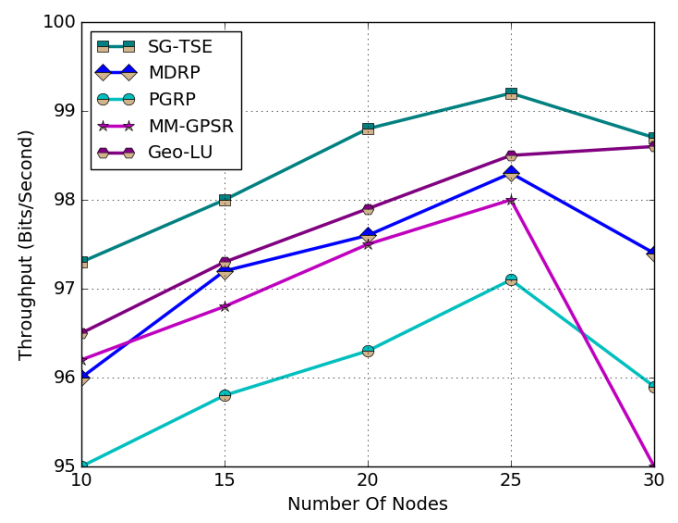

Fig. 4. Number of Nodes vs. Throughput.

Fig. 4 demonstrates the throughput comparison results of SG-TSE, MDRP, PGRP, MM-GPSR, and Geo-LU geographic routing protocols. All protocols increase the throughput from the point of 10 node densities to 25 node densities. For example, the SG-TSE obtains $97.3 \%$ and $99.2 \%$ of throughput under 10 and 25 number of nodes scenario, respectively. It is high in the range of $1.9 \%$. After point 25 , the throughput value of SG-TSE is decreased, as the link may fail due to high competition nodes. The SG-TSE minimizes the throughput by $0.5 \%$ after the point 25 number of nodes. However, the SGTSE attains better throughput performance than the other existing protocols. The main reason is that the SG-TSE selects the best $\mathrm{SH}$ nodes for data forwarding using multiple parameters that are position, speed, direction, and link quality. Thus, it maximizes the throughput even the network is highly congested. For instance, the SG-TSE improves the throughput by $1.3 \%, 2.3 \%, 1.1 \%$, and $0.8 \%$ than the existing MDRP, PGRP, MM-GPSR, Geo-LU when 10 numbers of vehicles are present in the network.

Fig. 5 shows the delay results of SG-TSE, MDRP, PGRP, MM-GPSR, and Geo-LU obtained with different numbers of nodes. The SG-TSE escalates the delay by adjusting the number of nodes from 10 to 30 . The main reason is that the nodes have to retransmit the packets frequently due to high packet loss under a high vehicle density setting. For example, the delay of SG-TSE is 0.35 seconds and 1.7 seconds for 10 and 30 nodes scenarios. However, the delay performance of SG-TSE is better than the other four geographic routing methods. The segment-based geographic router selection assists the SG-TSE to diminish the delay even the highly congested network. Also, the SG-TSE selects the best SH node by taking into account the position, speed, direction, and link quality parameters. Thus, it minimizes the delay in packet delivery and motivates the traffic lights for timely green phase activation, resulting in minimum $\mathrm{EV}$ arrival delay. For instance, the SG-TSE reduces the delay by $30 \%, 58.8 \%$, $78.1 \%$, and $63.2 \%$ than the MDRP, PGRP, MM-GPSR, and Geo-LU under 10 nodes scenario. It is varied by $69.6 \%$, $51.4 \%, 63 \%$, and $57.5 \%$ for 30 number of nodes scenario.

Fig. 6 illustrates the overhead comparison results of SGTSE, MDRP, PGRP, MM-GPSR, and Geo-LU by adjusting the number of nodes from 10 to 30 . All protocols increase the overhead by varying the node density from low to high. This is caused due to the utilization of a high number of control packets under the high-density scenario that maximizes the overhead in the network. For instance, the SG-TSE accomplishes 110 and 310 packets of overhead for 10 and 30 numbers of nodes, respectively. However, the SG-TSE diminishes the overhead than the MDRP, PGRP, MM-GPSR, and Geo-LU, as demonstrated in Fig. 6. The main reason is that the segment-based geographic routing along the EV route limits the control packets within the segment, resulting in minimum overhead. For example, when 30 nodes are present in the network, the SG-TSE, MDRP, PGRP, MM-GPSR, and Geo-LU attain 310, 350, 375, 410, and 375 packets of overhead, respectively.

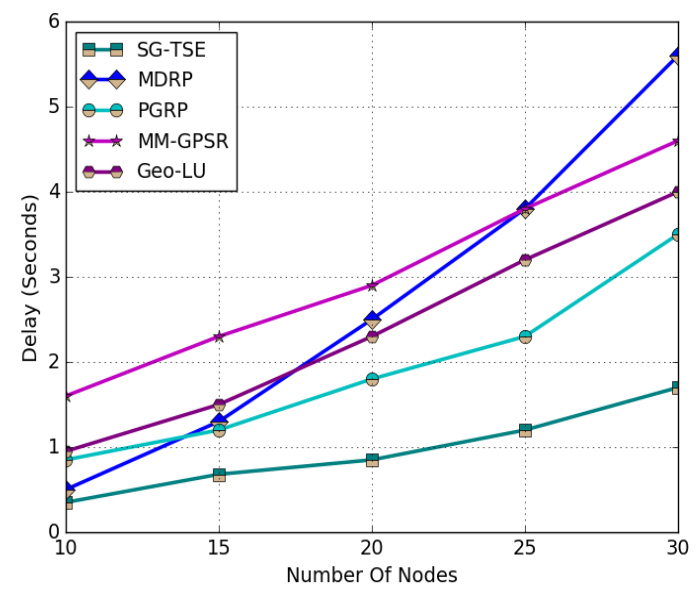

Fig. 5. Number of Nodes vs. Delay. 


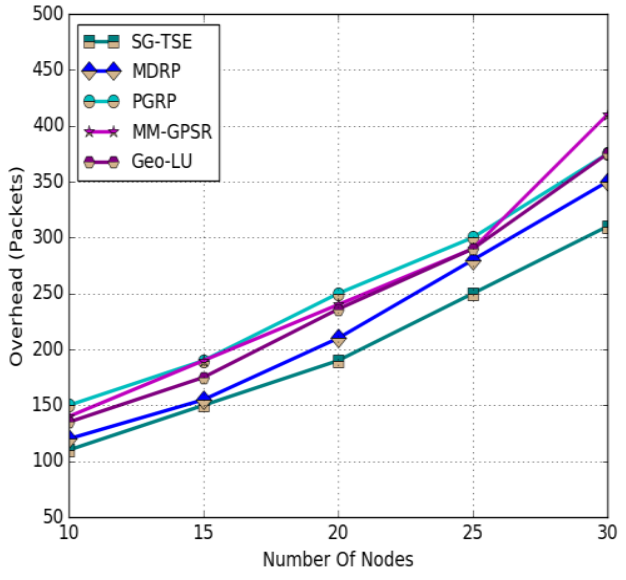

Fig. 6. Number of Nodes vs. Overhead.

The EV preemption speed results of SG-TSE obtained by varying the Number of Intersections (NoI) are depicted in Fig. 7. The EV preemption speed is diminished when varying the vehicle density from 10 to 30 . The main reason is that the traffic controller requires a long time to pre-clear the vehicles under a high vehicle density scenario, impacting EV preemption speed. For instance, the speed of EV is 60 and 55 $\mathrm{Km} / \mathrm{Hr}$ for 10 and 30 node density scenarios under one intersection. The SG-TSE design considers the NoI into account, and there is a need to recalculate the minimum detection distance when more than one intersection is presented along the EV route. Thus, it creates some impact on EV speed. For instance, the speed of EV is diminished by $8.3 \%$ for 20 nodes with two NoI scenarios. However, the timely green phase activation with minimum detection distance measurement in SG-TSE speeds up the EV at intersections rapidly and minimizes the arrival delay even the network is congested.

Fig. 8 obtains the PDR comparative results of SG-TSE, MDRP, PGRP, MM-GPSR, and Geo-LU by varying the vehicle speed from 20 to $60 \mathrm{Km} / \mathrm{hr}$. The results show that the SG-TSE decreases the PDR by adjusting the vehicle speed from 20 to $60 \mathrm{Km} / \mathrm{Hr}$. It is caused due to the frequent link disconnections of high-speed vehicles in the network. For example, the SG-TSE accomplishes $99 \%$ and $97.1 \%$ of PDR when the nodes move with speed $20 \mathrm{Km} / \mathrm{Hr}$ and $60 \mathrm{Km} / \mathrm{Hr}$, respectively. However, the SG-TSE obtain better PDR results by selecting the $\mathrm{SH}$ nodes with multi-criteria information like position, speed, direction, and link quality than the existing protocols. Thus, it effectively handles the frequent link disconnections and boosts the PDR even when vehicles move at high speed. For instance, the SG-TSE increases the PDR by $0.3 \%, 1.1 \%, 4.1 \%$, and $2.6 \%$ than the existing MDRP, PGRP, MM-GPSR, and Geo-LU protocols under a high vehicle speed scenario of $60 \mathrm{Km} / \mathrm{hr}$.

Fig. 9 shows the delay results of SG-TSE, MDRP, PGRP, MM-GPSR, and Geo-LU protocols. The results are accomplished by adjusting the speed values of the vehicles from 20 to $60 \mathrm{Km} / \mathrm{hr}$. All protocols increase the delay by varying the vehicle speed from low to high. The main reason is that the nodes have to retransmit the packets frequently due to link disconnections. For example, the delay of SG-TSE is
0.5 seconds and 2.3 seconds for 20 and $60 \mathrm{Km} / \mathrm{Hr}$ of vehicle speeds, respectively. However, the delay performance of SGTSE is better than the other existing MDRP, PGRP, MMGPSR, and Geo-LU protocols. For instance, the SG-TSE reduces the delay by $54.5 \%, 37.5 \%, 58.3 \%$, and $66.7 \%$ than the MDRP, PGRP, MM-GPSR, and Geo-LU when the vehicles move with $20 \mathrm{Km} / \mathrm{Hr}$ speed. The reason is that the multi-criteria-based SH router selection assists the SG-TSE to diminish the delay even the highly congested network. Thus, it maximizes the green time activation accuracy. Moreover, the SG-TSE minimizes the negative impact on normal vehicles owing to EV preemption by activating the green phase at the right time.

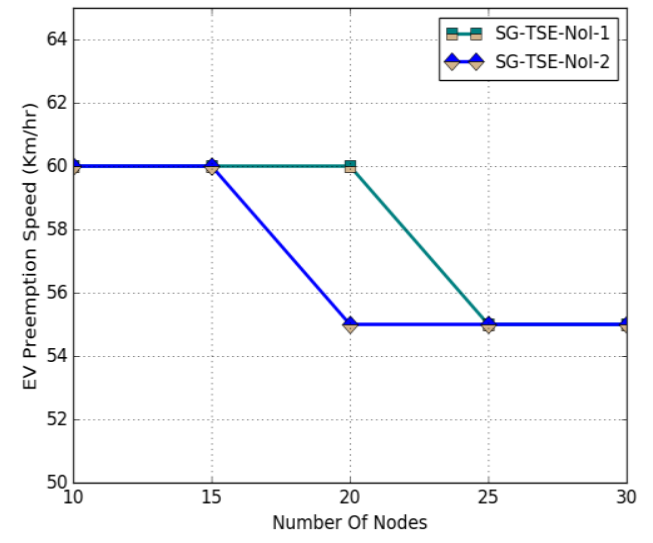

Fig. 7. Number of Nodes vs. EV Preemption Speed.

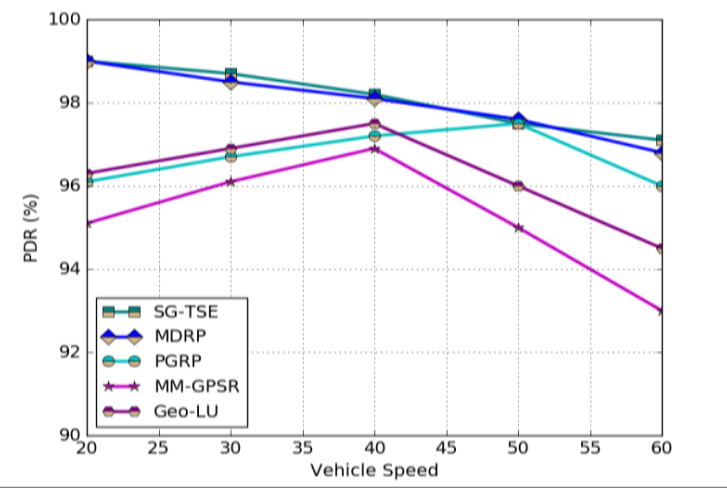

Fig. 8. Vehicle Speed vs. PDR

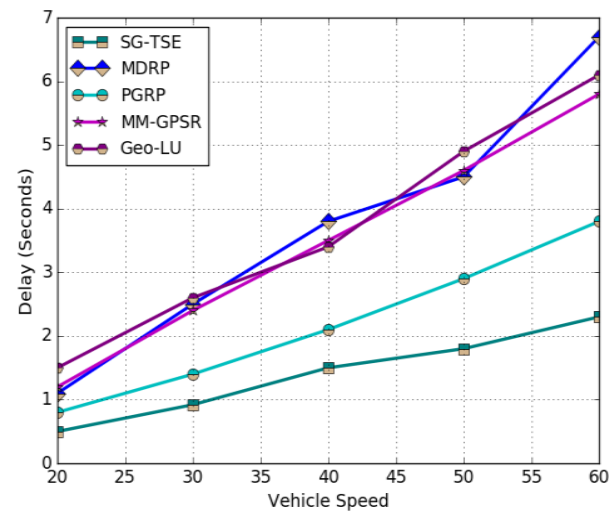

Fig. 9. Vehicle Speed vs. Delay. 


\section{CONCLUSION}

In this paper, a novel EV preemption method SG-TSE has been proposed to reduce the negative impacts of normal vehicles on EV speed. To achieve the objective, the SG-TSE includes two mechanisms that are SGR and DTSE. By dividing the approaching route into multiple segments and performing segment-based geographic routing, the SGR instructs the traffic light controller about EV arrival. The minimum detection distance measurement based green phase adjustment in DTSE reduces the negative impacts of normal traffic on EV speed and neglects the EV preemption negative effects on normal traffic. Moreover, the NS-2 based simulation depicts the effectiveness of the proposed SG-TSE with different performance metrics like PDR, overhead, delay, throughput, and EV preemption speed. From the results, the EV maintains its speed in the approaching lane without disturbing the normal traffic conditions.

\section{REFERENCES}

[1] Zeadally, S., Hunt, R., Chen, YS. et al, "Vehicular ad hoc networks (VANETS): status, results, and challenges", Telecommun Syst, pp. 217241, 2012, https://doi.org/10.1007/s11235-010-9400-5.

[2] Michael Lee, Travis Atkison, "VANET applications: Past, present, and future, Vehicular Communications", Vol. 28, 2021.

[3] V. Paruchuri, "Adaptive Preemption of Traffic for Emergency Vehicles", UKSim-AMSS 19th International Conference on Computer Modelling \& Simulation (UKSim), pp. 45-49, 2017, doi: 10.1109/UKSim.2017.34.

[4] Almuraykhi, K. M., \&Akhlaq, M, "STLS: Smart Traffic Lights System for Emergency Response Vehicles", International Conference on Computer and Information Sciences (ICCIS), 2019.

[5] W. Min, L. Yu, P. Chen, M. Zhang, Y. Liu and J. Wang, "On-Demand Greenwave for Emergency Vehicles in a Time-Varying Road Network With Uncertainties", in IEEE Transactions on Intelligent Transportation Systems, vol. 21, no. 7, pp. 3056-3068, July 2020, doi: 10.1109/TITS.2019.2923802.

[6] R. Anil, M. Satyakumar and A. Salim, "Emergency Vehicle Signal Preemption System for Heterogeneous Traffic Condition : A Case Study in Trivandrum City", 2019 4th International Conference on Intelligent Transportation Engineering (ICITE), 2019, pp. 306-310, doi: 10.1109/ICITE.2019.8880151.

[7] SouaadBoussoufa-Lahlah, FouziSemchedine, and LouizaBoualloucheMedjkoune, "Geographic routing protocols for Vehicular Ad hoc NETworks (VANETs): A survey", Vehicular Communications, Volume $11,2018$.

[8] Karimi, R., \&Shokrollahi, S, "PGRP: Predictive geographic routing protocol for VANETs", Computer Networks, 2018.

[9] Yang, X., Li, M., Qian, Z., \& Di, T, "Improvement of GPSR Protocol in Vehicular Ad Hoc Network", IEEE Access, pp. 39515-39524, 2018.

[10] Alzamzami, O., \&Mahgoub, I, "Link Utility Aware Geographic Routing for Urban VANETs using Two-Hop Neighbor Information”, Ad Hoc Networks, 2020.

[11] MeenaakshiSundhari, R. P., Murali, L., Baskar, S., \& Shakeel, P. M, "MDRP: Message dissemination with re-route planning method for emergency vehicle information exchange", Peer-to-Peer Networking and Applications, 2020.

[12] P. Devi and S. Anila, "Intelligent Ambulance with Automatic Traffic Control", 2020 International Conference on Computing and Information Technology (ICCIT-1441), pp. 1-4, 2020, doi: 10.1109/ICCIT144147971.2020.9213796.
[13] Subash Humagain, Roopak Sinha, Edmund Lai \& Prakash Ranjitkar, "A systematic review of route optimisation and pre-emption methods for emergency vehicles", Transport Reviews, Vol. 40, No. 1, pp. 35-53, 2020, DOI: 10.1080/01441647.2019.1649319.

[14] Oza, P., \&Chantem, T, "Timely and Non-Disruptive Response of Emergency Vehicles: A Real-Time Approach", 29th International Conference on Real-Time Networks and Systems, 2021.

[15] Wenwen Kang, Gang Xiong, YishengLv, Xisong Dong, Fenghua Zhu, \&Qingjie Kong, "Traffic signal coordination for emergency vehicles", 17th International IEEE Conference on Intelligent Transportation Systems (ITSC), 2014, doi:10.1109/itsc.2014.6957683.

[16] Ziling Zeng; Wen Yi; Shuaian Wang; and Xiaobo Qu,"Emergency Vehicle Routing in Urban Road Networks with Multistakeholder Cooperation", Journal of Transportation Engineering, Part A: Systems, Vol. 147, No. 10, 2021.

[17] S. Humagain and R. Sinha, "Dynamic Prioritization of Emergency Vehicles For Self-Organizing Traffic using VTL+EV", IECON 2020 The 46th Annual Conference of the IEEE Industrial Electronics Society, pp. 789-794, 2020, doi: 10.1109/IECON43393.2020.9254313.

[18] A. S. Eltayeb, H. O. Almubarak and T. A. Attia, "A GPS based traffic light pre-emption control system for emergency vehicles", International Conference on Computing, Electrical and Electronic Engineering (ICCEEE), pp. 724-729, 2013, doi: 10.1109/ICCEEE.2013.6634030.

[19] Hamed Noori, Liping Fu, and SajadShiravi, "A Connected Vehicle Based Traffic Signal Control Strategy for Emergency Vehicle Preemption", 2016.

[20] Asaduzzaman, M., \&Vidyasankar, K, "A Priority Algorithm to Control the Traffic Signal for Emergency Vehicles", IEEE 86th Vehicular Technology Conference (VTC-Fall), 2017, doi:10.1109/vtcfall.2017. 8288364.

[21] Wu, J., Kulcsár, B., Ahn, S., \& Qu, X, "Emergency vehicle lane preclearing: From microscopic cooperation to routing decision making", Transportation Research Part B: Methodological, pp. 223-239, 2020, doi:10.1016/j.trb.2020.09.011.

[22] VítObrusník, Ivo Herman, ZdeněkHurák, "Queue discharge-based emergency vehicle traffic signal preemption", The research was funded by Technology Agency of the Czech Republic within the program Epsilon, the project TH03010155.,IFAC-PapersOnLine, Vol. 53, No. 2, 2020.

[23] Mu, H., Song, Y., \& Liu, L, "Route-Based Signal Preemption Control of Emergency Vehicle", Journal of Control Science and Engineering, 2018, 1-11. doi:10.1155/2018/1024382.

[24] Rathore, N., Jain, P. K., \&Parida, M, “A Routing Model for Emergency Vehicles using the Real Time Traffic Data". IEEE International Conference on Service Operations and Logistics, and Informatics (SOLI), 2018.

[25] Younes, M.B., Boukerche, A, "An efficient dynamic traffic light scheduling algorithm considering emergency vehicles for intelligent transportation systems", Wireless Networks, pp. 2451-2463, 2018.

[26] Louati, A., Elkosantini, S., Darmoul, S., \&Louati, H, "Multi-agent preemptive longest queue first system to manage the crossing of emergency vehicles at interrupted intersections", European Transport Research Review, Vol. 10, No. 2, 2018.

[27] Shaaban, K., Khan, M. A., Hamila, R., \& Ghanim, M, "A Strategy for Emergency Vehicle Preemption and Route Selection", Arabian Journal for Science and Engineering, 2019.

[28] Talebi, M., \& Sabaei, M, "Smartly, reduce the latency of high-priority vehicles using loT technology" In IEEE 29th Iranian Conference on Electrical Engineering (ICEE), pp. pp. 514-520, 2021.

[29] Kamble, Shridevi J., and Manjunath R. Kounte. "On Road Intelligent Vehicle Path Predication and Clustering using Machine Learning Approach." 2019 Third International conference on I-SMAC (IoT in Social, Mobile, Analytics and Cloud)(I-SMAC). IEEE, 2019. 
[30] M. V, A. V and S. Allirani, "Smart Emergency Vehicle Plan with App Monitoring and Centralized Network", 6th International Conference on Communication and Electronics Systems (ICCES), pp. 1-5, 2021.

[31] Obrusník, V., Herman, I., \& Hurák, Z, "Queue discharge-based emergency vehicle traffic signal preemption", IFAC-PapersOnLine, Vol. 53, No. 2, pp. 14997-15002, 2020.
[32] Kamble, Shridevi Jeevan, and Manjunath R. Kounte. "Machine learning approach on traffic congestion monitoring system in internet of vehicles." Procedia Computer Science 171 (2020): 2235-2241.

[33] SEO, J., KIM, D., KANG, S., LEE, J., \& LEE, S, "Development of Signal Priority Algorithm considering a Recovery Time for Emergency Vehicle", 2021. 